\title{
Changes in the Diversity of Human Skin Microbiota to Cosmetic Serum Containing Prebiotics: Results from a Randomized Controlled Trial
}

\author{
Ki-Bae Hong ${ }^{1}$, Yang Hee Hong ${ }^{2}$, Eun Young Jung ${ }^{3}$, Kyungae Jo ${ }^{4}$ : and Hyung Joo Suh ${ }^{1, *}$ \\ 1 Department of Integrated Biomedical and Life Sciences, Graduate School, Korea University, \\ Seoul 02841, Korea; kibae.hong@gmail.com \\ 2 Department of Beauty Art, Suwon Women's University, Suwon 16632, Korea; hongyh@swc.ac.kr \\ 3 Department of Home Economic Education, Jeonju University, Jeonju 55069, Korea; jjjj@jj.ac.kr \\ 4 BK21Plus, College of Health Science, Korea University, Seoul 02841, Korea; kyungae11@korea.ac.kr \\ * Correspondence: suh1960@korea.ac.kr
}

Received: 3 July 2020; Accepted: 11 August 2020; Published: 17 August 2020

\begin{abstract}
Prebiotic treatment may rebalance the skin microbiota by regulating the growth of harmful and beneficial microorganisms. In this randomized, double-blind, placebo-controlled clinical trial $(\mathrm{N}=60)$, we evaluated the effects of a cosmetic serum containing galacto-oligosaccharides (GOS) on the balance of the skin microbiota by measuring various skin parameters. The skin water-holding capacity between the control (ND) and experimental (NF) groups was significantly different after 8 weeks of serum treatment $(p<0.05)$. Similarly, changes in transepidermal water loss (TEWL) and the erythema index in the ND and NF groups were significantly different $(p<0.05)$. Furthermore, the wrinkle depth and Staphylococcus aureus population decreased in the NF group compared with those in the ND group $(p<0.05)$. The mean form factor, Shannon index, and Pediococcus population were significantly increased in the post-NF group compared with those in the post-ND group $(p<0.05)$. Finally, in the ND group, water-holding capacity was positively correlated with Enhydrobacter, whereas Enterobacteriaceae was negatively correlated with TEWL in the NF group. These results suggest that GOS inhibit the growth of harmful skin microbes and increase the population of beneficial microbes.
\end{abstract}

Keywords: prebiotics; GOS; skin microbiota; Staphylococcus aureus

\section{Introduction}

The skin acts as a barrier that defends against potential hazards and toxins from the surrounding environment [1]. The skin is the point of contact between the external and internal environment and allows the growth of various microorganisms, including fungi, bacteria, viruses, and small larvae [2]. The skin microbiota plays a major role in the suppression of pathogenic species and regulation of skin proteins, free fatty acids, and lipid-rich substances [3]. The skin combines physical and biological factors to create a microbial habitat, and factors that affect symbiosis between humans and microorganisms may impair skin barrier functions, leading to infections [4].

The skin microbiota produces various metabolites, such as aureusimine A and B, which directly induce differential gene expression in human keratinocytes [5-7]. The functions of these metabolites are reported to be involved in the expression of the virulence factor gene in $S$. aureus, inhibition of protease in eukaryotic cells, and interspecies bacterial communication [7]. It is necessary to use antimicrobial agents that inhibit the growth of harmful bacteria, such as Propionibacterium acnes, or create an environment that favors the growth of beneficial microorganisms, such as Staphylococcus epidermis. Prebiotic treatment can cause specific changes in the microbial diversity and colonization that could rebalance the skin microbiota [8]. Several attempts have been directed towards the use of prebiotic 
cosmetics to balance the composition of skin microbiota; however, the effects of galacto-oligosaccharides (GOS), a prebiotic, on skin microbiota diversity are still unknown $[8,9]$.

GOS are indigestible food components that could improve the health of the host by selectively increasing the population of beneficial microorganisms [10]. In our previous study [11], oral administration of GOS (2 g/day) decreased the trans-epidermal water loss (TEWL), increased the water-holding capacity, and reduced the area of wrinkles on the skin. Further, GOS cause changes in intestinal microbiota and the mucosal immune system, which influence the skin. In addition, like prebiotics, the use of cosmetic formulation containing sugar and beta-glucan has been reported to improve various human skin conditions such as skin dehydration and wrinkle formation. However, whether GOS can have such beneficial effects on skin conditions remains unknown [12,13].

In the present clinical trial, a cosmetic serum containing GOS was directly applied to the facial skin to investigate the changes in the microorganisms on the alar crease (side of the nose). We evaluated the 8-week treatment with GOS-containing cosmetic serum is effective in improving various skin parameters and the population of beneficial skin microbes.

\section{Materials and Methods}

\subsection{Cosmetic Serum Formulation}

The cosmetic serum (Formulation NF) was prepared using commercially available PEG-60 hydrogenated castor oil $(0.3 \%)$, cetyl ethylhexanoate $(0.5 \%)$, arginine $(0.17 \%)$, hydroxyethylcellulose $(0.1 \%)$, carbomer \#941 $(0.07 \%)$, carbomer \#940 $(0.1 \%)$, ethylhexylglycerin $(0.05 \%)$, phenoxyethanol $(0.4 \%)$, disodium ethylenediaminetetraacetic acid (EDTA, 0.03\%), GOS $(7.0 \%)$, and deionized water (to reach $100 \%$ ). Serum without GOS (Formulation ND) was used as control. The serum samples were mixed at room temperature. Formulation ND and NF were obtained from Phytacoid Inc., Suwon, Korea. GOS used in the cosmetic serum was donated by Neo Crema Co., Ltd. (Seoul, Korea).

\subsection{Subjects and Sampling}

The subjects of this study were healthy women in their 40s and 60s, and all subjects signed a clinical trial agreement after receiving a detailed explanation on the purpose and contents of the study. There were no dropouts, and a total of 60 measurement results were used for statistical analysis. None of the subjects had a history of skin infections, skin diseases, or other chronic diseases and were in good health and did not receive antibiotics or antifungal agents for more than 1 month. According to Fitzpatrick classification, the subjects were classified as skin types II and III [14] and were instructed to disuse hygiene products such as cosmetics, moisturizers, and antiperspirant deodorants for $12 \mathrm{~h}$ prior to sampling. In a randomized controlled trial, 60 women were screened and received either the placebo (formulation ND; $N=30$; the median age: 50.33; 40s: 19; 50s: $5 ; 60 \mathrm{~s}: 6$ ) or cosmetic serum (formulation NF; $\mathrm{N}=30$; the median age: $50.43 ; 40$ s: 20 ; 50s: 5; 60s: 5) for 8 weeks. All subjects applied a total of $1.0 \mathrm{~mL}$ of the sample to their faces twice a day $(0.5 \mathrm{~mL}$ in the morning and at night) for 8 weeks, and skin properties were evaluated. For skin microbiota analysis, subjects were investigated by the researcher who proceeded the study and collected samples. Subjects who used medicinal soaps and facial cleanser $6 \mathrm{~h}$ before obtaining analytical samples $(\mathrm{n}=32)$, and those who did not wish to analyze their composition of skin microbiota $(n=12)$ were not included in the experiment. Additionally, only the subjects who provided written consent were analyzed for the skin microbiota and were randomly divided into groups $(n=16)$. Skin samples for skin microbiota analysis were obtained from the alar crease (side of the nose) with a sterile cotton swab. The head of each swab was cut-off and stored at $4{ }^{\circ} \mathrm{C}$ until DNA extraction. The study was approved by the Jeonju University Institutional Review Board (jjIRB-190115-HR-2019-0106) in Korea and was conducted in accordance with the ethical standards of 1964 Declaration of Helsinki. 


\subsection{Skin Assessments}

The surface and TEWL of the skin were measured using the Corneometer CM825 (Courage and Khazaka electronic GmbH, Cologne, Germany) and Tewameter TM300 (Courage and Khazaka electronic $\mathrm{GmbH}$, Cologne, Germany). Melanin index and erythema index of the skin were measured using the Mexameter MX18 (Courage and Khazaka electronic GmbH, Cologne, Germany). To measure wrinkles from the corner of the eye (Crow's feet), the skin replicas were obtained before and after the experiment using the silicon solution Silflo (Flexico Ltd., London, England). All simulated plates were analyzed for six different wrinkle parameters: total wrinkle area, percent of wrinkle area, number of wrinkles, total length, wrinkle depth, and mean form factor, using the Visioline VL650 (Courage and Khazaka electronic GmbH, Cologne, Germany).

\subsection{DNA Extraction, Amplification, and Sequencing}

DNA from the skin microbiota was extracted from the cotton swab with i-genomic Clinic DNA Extraction Kit (iNtRON Biotech, Sung-Nam, Korea) according to the manufacturer's protocol. The $16 \mathrm{~S}$ rRNA amplicon libraries were constructed by slightly modifying the protocol of Illumina 16S V3 V4 Amplicon (Illumina, San Diego, CA, USA). The $16 \mathrm{~S}$ rRNA V3 V4 regions were amplified by first polymerase chain reaction (PCR-I) as follows: $2 \mathrm{~min}$ at $95^{\circ} \mathrm{C}, 25$ cycles of $30 \mathrm{~s}$ at $95{ }^{\circ} \mathrm{C}, 30 \mathrm{~s}$ at $55^{\circ} \mathrm{C}$, and $30 \mathrm{~s}$ at $72{ }^{\circ} \mathrm{C}$, and $10 \mathrm{~min}$ at $72{ }^{\circ} \mathrm{C}$. The PCR products were purified using the Agencourt AMPure XP Beads (Beckman Coulter, Inc., Pasadena, CA, USA) and then quantified. A second PCR (PCR-II) was conducted using a quality-controlled PCR product as a template and the Illumina dual-index adapter sequences. The PCR-II cycling conditions were as follows: $3 \mathrm{~min}$ at $95^{\circ} \mathrm{C}, 8$ cycles of $30 \mathrm{~s}$ at $95{ }^{\circ} \mathrm{C}, 30 \mathrm{~s}$ at $55^{\circ} \mathrm{C}$, and $30 \mathrm{~s}$ at $72{ }^{\circ} \mathrm{C}$, and $5 \mathrm{~min}$ at $72{ }^{\circ} \mathrm{C}$. Next, amplicons were purified with Agencourt AMPure XP Beads (Beckman Coulter, Inc., Pasadena, CA, USA), and their quality and quantity were assessed with GX caliper HS DNA 1000 (PerkinElmer, Waltham, MA, USA) and the NanoQuant pro200 instrument (Tecan, Mannedorf, Switzerland). The final amplicon libraries were sequenced on an Illumina MiSeq using the v3 600 cycle kit and 301 base-paired end chemistry.

\subsection{Bioinformatic Analysis Using $16 S$ rRNA Sequences}

The sequence data were analyzed using the QIIME 2 software [15]. Prior to sequencing, quality filtering was performed for terminal trimming and to remove low-quality and non-target sequences $(<0.2 \mathrm{~kb})$ and ambiguous reads. To eliminate homopolymers, the sequence error rate was minimized using the QIIME pipeline [16]. Representative sequence sets were selected and clustered using UCLUST at a similarity level of $97.0 \%$ into OTUs and were then analyzed. Processed sequences were aligned with PyNAST using Greengenes Database (gg_97_otus_4-feb2011.fasta) as a reference [17], and taxonomy was assigned using the ribosomal database project classifier [18] with a minimum confidence score of 0.8. Richness, Shannon Index, and ACE index were included in the alpha-diversity analysis using MOTHUR [19]. Beta-diversity within and between groups was analyzed using UniFrac metric distances. These results were analyzed with PCoA [20].

\subsection{Statistical Analysis}

Data are presented as mean \pm standard error. Data were analyzed using one-way ANOVA with Tukey's post-hoc test using SPSS (v16.0, Chicago, IL, USA). A comparison between two groups was performed using Student's t-test. Correlation between skin microbial composition (OTU abundance) and the mean of skin parameters in the same group was confirmed using Pearson's correlation (SPSS version 19.0). To analyze differences in alpha-diversity and relative abundance in bacterial genera, Kruskal-Wallis test was performed. The differences in beta-diversity were analyzed with ANOSIM and permutational multivariate analysis of variance (PERMANOVA) tests. A $p$-value $<0.05$ was considered significant. 


\section{Results}

3.1. Changes in the Water Holding Capacity of the Skin Surface and TEWL by Prebiotics-Containing Cosmetic Serum Treatment

The delta-values representing of changes in the Corneometer value relative to the initial value are shown in Figure 1. The water-holding capacity was proportional to the increase in the Corneometer values. The values of the control (ND) and experimental (NF) groups at week 0 were 73.26 AU and 69.20 AU, respectively. The skin water-holding capacity decreased gradually after initiating the experimental period but increased slightly after 4 weeks. The skin water-holding capacity of the NF group was significantly higher after 8 weeks of treatment than that of the ND group $(p<0.05)$ (Figure 1A). TEWL refers to the moisture evaporating from the skin and is used as an indicator of the skin barrier function in the stratum corneum. The lower the TEWL, the lower the water loss and the better the skin's defense ability. The TEWL values of the subjects at the start of the experiment were $13.84 \mathrm{~g} / \mathrm{h} / \mathrm{m}^{2}$ and $14.40 \mathrm{~g} / \mathrm{h} / \mathrm{m}^{2}$ in the ND and NF groups, respectively. The initial TEWL value in the ND group was significantly lower than that at 6 to 8 weeks. Moreover, the delta-values, indicating the change in TEWL in the NF groups, were significantly lower between weeks 4 and 8 than those in the ND group (Figure 1B). These results indicate that GOS can help maintain skin barrier function.

A

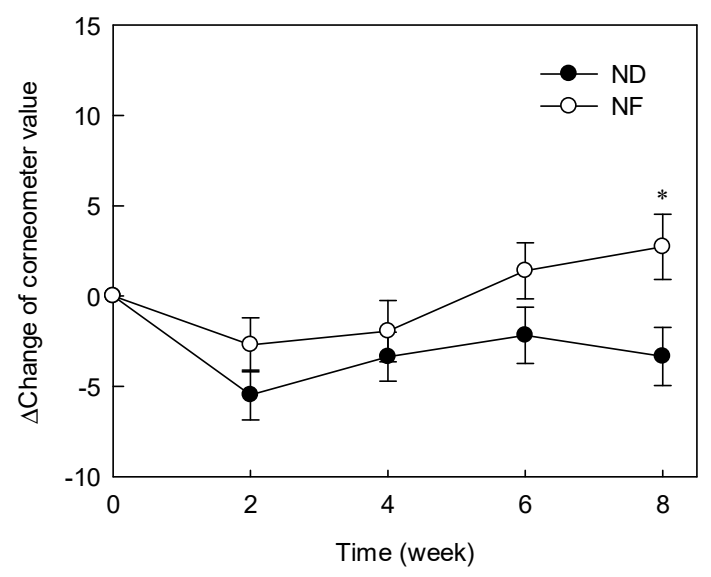

$\mathbf{B}$

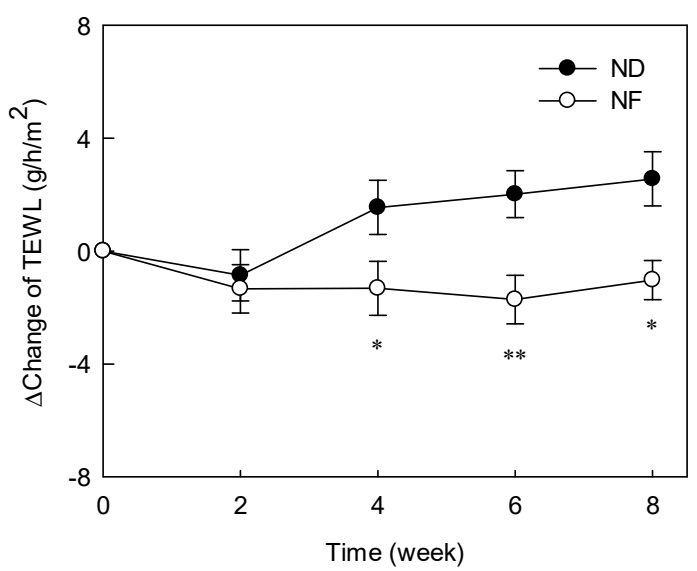

Figure 1. Effects of serum cosmetics containing galacto-oligosaccharides (GOS) on water holding capacity (A) and transepidermal water loss (TEWL) (B) from baseline after 8 weeks of treatment in healthy adults. The asterisks indicate significant differences $\left({ }^{*} p<0.05\right.$ and $\left.{ }^{* *} p<0.01\right)$ between the NF and the ND groups at the indicated week. The $\mathrm{p}$ of two-sided independent $\mathrm{t}$-test method was displayed, and all data are reported as mean \pm standard error of the mean. ND (control serum); NF (cosmetic serum containing GOS).

\subsection{Changes in Skin Pigments by Prebiotics-Containing Cosmetic Serum Treatment}

Melanin and erythema pigments were measured during the GOS-containing cosmetic serum treatment period (Figure 2). The melanin pigment decreased during the experimental period in both ND and NF groups. The melanin index of the ND group decreased after 2 weeks, but the difference was not statistically significant. There were also no significant differences between the delta values between the two groups (Figure 2A). Further, the erythema index of the NF group was not significantly changed during the experimental period compared with that of the ND group but was statistically significantly decreased after 4 weeks of treatment compared to the base line (week 0 ). Figure $2 \mathrm{~B}$ shows the difference in delta-values between the two groups. The difference of the erythema index between NF and ND groups was statistically significant at week $8(p<0.05)$. GOS-containing cosmetic serum was effective in reducing the erythema index but had no significant effect on the melanin pigment. 
A

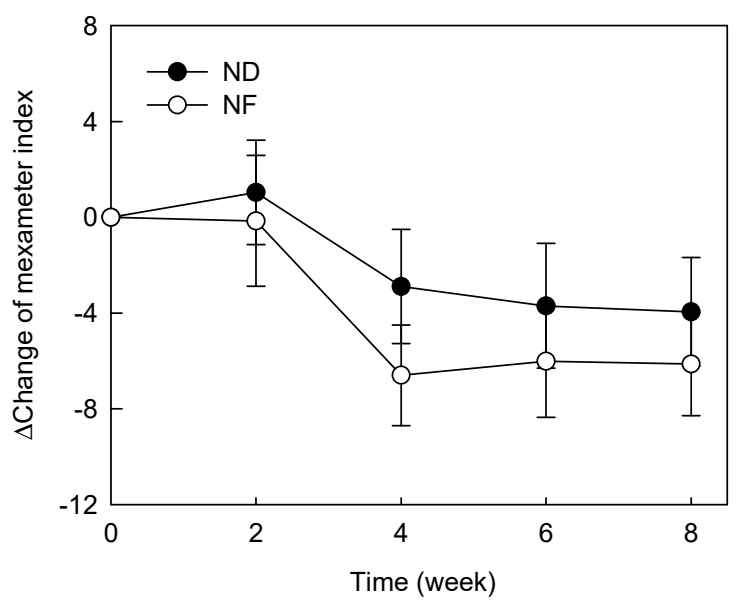

B

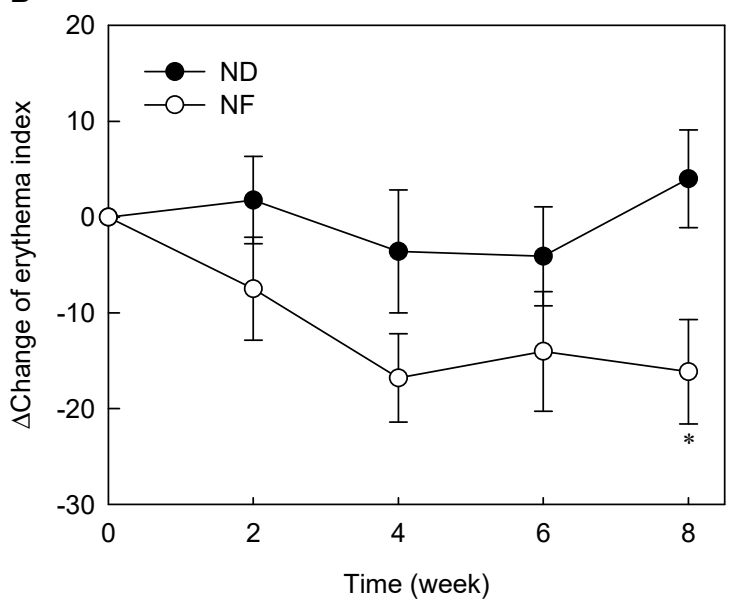

Figure 2. Effects of serum cosmetics containing GOS on melanin index (A) and erythema index (B) from baseline after 8 weeks of treatment in healthy adults. The asterisks indicate significant differences $\left({ }^{*} p<0.05\right)$ between the NF and the ND groups at the indicated week. The p of two-sided independent $\mathrm{t}$-test method was displayed, and all data are reported as mean \pm standard error of the mean. ND (control serum); NF (cosmetic serum containing GOS).

\subsection{Changes in the Skin Wrinkles Using Replica by Prebiotics-Containing Cosmetic Serum Treatment}

The changes in the wrinkle-related factors in the NF and ND groups before and after the serum application were measured using a replica and Visioline. The wrinkle area after the experiment increased in the NF group but was not significantly different when compared with that in the ND group. Similarly, the number, total length, and depth of wrinkles in the NF group decreased after the experiment but was not significantly different. The mean form factor, which is the average ratio of the width and length of all shadows considered wrinkles in the Visioline analysis, was significantly increased in the NF group compared with that in the ND group (Figure 3). Visioline documentation states, "This element is always between 0 (seamless circle) and 1 (seamless line). The closer the form factor is to 1 , the more effective is the anti-wrinkle treatment" [21].

\subsection{Changes in the Water Holding Capacity of the Skin Surface and TEWL by Prebiotics-Containing Cosmetic Serum Treatment}

The 16S rRNA gene amplificons were subjected to MiSeq sequencing (Illumina, San Diego, CA, USA). We used a dataset of passed filter paired-end raw sequence reads and detected 15,132 operational taxonomic units (OTUs) in all groups. In total, 2810 and 3020 isolates were obtained from eight individuals in the pre-ND and pre-NF groups, respectively, and 152-991 (average, 351.3 \pm 94.7) and 259-594 (average, $377.5 \pm 39.5$ ) clones were analyzed for each subject from pre-ND and pre-NF groups, respectively. Actinobacteria, Firmicutes, and Proteobacteria were identified as the main phyla before treatment. Proteobacteria accounted for $40.75 \%$ and $41.63 \%$ of all isolates in the pre-ND and pre-NF groups, respectively; Firmicutes, for $37.3 \%$ and $32.18 \%$; and Actinobacteria, for $16.46 \%$ and $18.82 \%$ (Supplementary 1). Proteobacteria accounted for $34.37 \%$ and $37.94 \%$ of all isolates in the post-ND and post-NF groups, respectively; Firmicutes, for $40.43 \%$ and $34.27 \%$; and Actinobacteria, for $19.56 \%$ and $20.09 \%$ (Supplementary 2). After 8 weeks of treatment, the proportion of Firmicutes increased, while that of Proteobacteria decreased. In pre-ND and post-ND groups, the main genera observed were Staphylococcus (22.08\% and 26.33\%, respectively) and Cutibacterium (11.46\% and 13.70\%, respectively). 

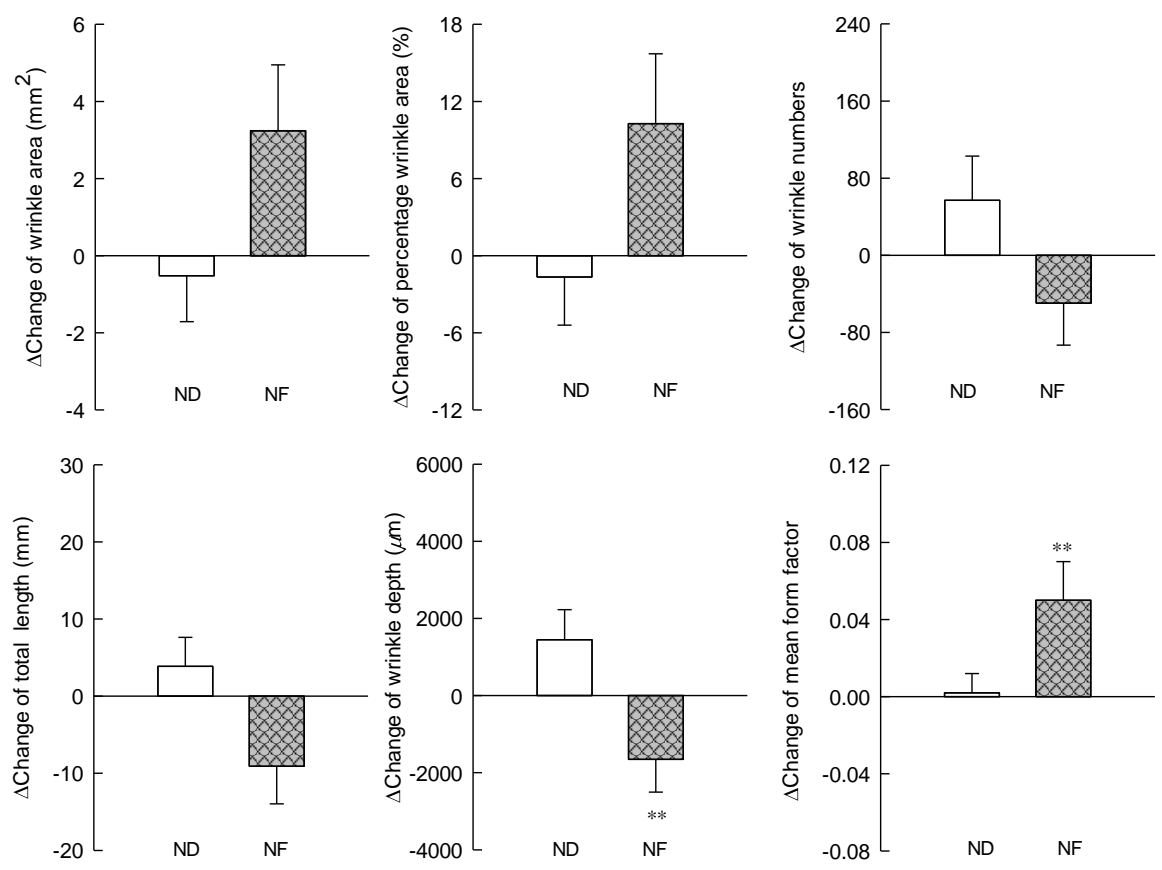

Figure 3. Effects of serum cosmetics containing GOS on wrinkle index from baseline after 8 weeks of treatment in healthy adults. The asterisks indicate significant differences $\left({ }^{* *} p<0.01\right)$ between the NF and the ND groups at the indicated week. The p of two-sided independent $t$-test method was displayed, and all data are reported as mean \pm standard error of the mean. ND (control serum); NF (cosmetic serum containing GOS).

\subsection{Microbial Diversity Within and between the Groups}

Alpha- and beta- diversity represent microbial diversity within or between groups, respectively. For estimation of the species richness, we calculated the number of species expected from the abundance-based coverage estimator (ACE) index. In this assessment, the species richness increased in both the post-ND and post-NF groups compared with that in pre-ND and pre-NF groups; however, no significant difference in species richness was observed between the post- and pre-treatment groups. Moreover, inter-group differences in species richness were absent (Figure 4A). The post-NF group exhibited higher species diversity than the post-ND group (Figure 4A). These results suggest that GOS-containing serum affected diversity, rather than species richness (Figure 4). Further, Shannon index was used to assess species diversity (alpha-diversity) within or between the groups (Figure 4B). Although Shannon index of the post-treatment groups showed no significant difference as compared with that of the pre-treatment groups, a significant difference in species diversity was reported between the post-ND and post-NF groups ( $p<0.05$; Figure 4B). The addition of GOS resulted in an increase in species diversity, which was significantly different from the serum base (post-ND). Our results showed that, statistically, the bacterial richness on the facial skin of the post-NF group was not significantly different compared with that of the post-ND group. However, the diversity of the bacterial communities was found to be caused by the application of GOS-containing cosmetic serum. 

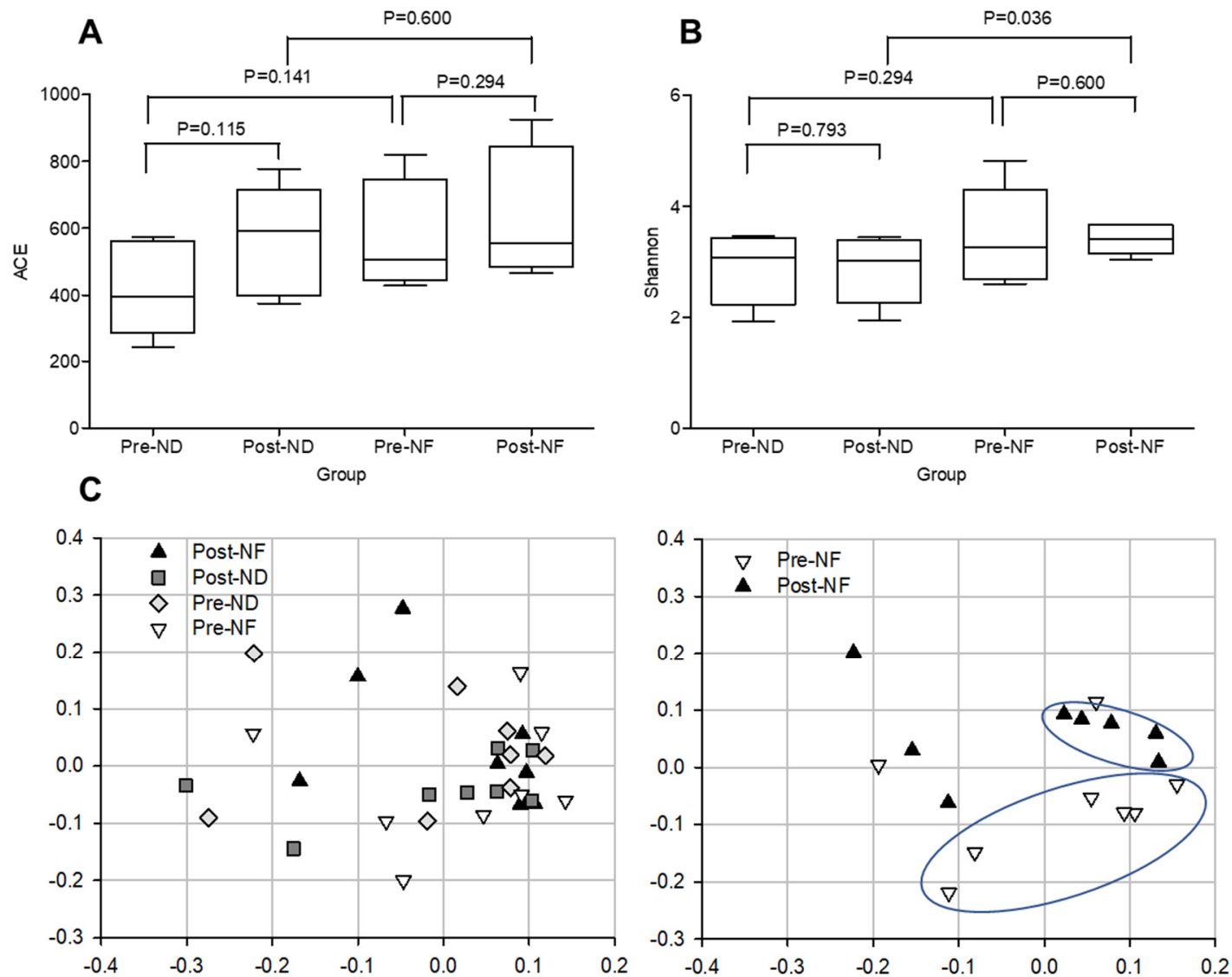

Figure 4. Species richness (ACE index), species diversity (Shannon index), and beta-diversity (PCoA) of species within and between groups. (A) Species richness (ACE index), (B) species diversity (Shannon index), (C) beta-diversity (PCoA). ND (control serum); NF (cosmetic serum containing GOS).

Principal coordinate analysis (PCoA) was used to compare the beta-diversity profiles across different treatment groups (Figure 4C). According to PcoA (Figure 4C), the pre-NF group was mainly distributed in quadrants 3 and 4, while the post-NF group was distributed in quadrants 1 and 2 . The PCoA plot indicated an obvious and a statistically significant separation between the four groups, and the center point coordinate of the ellipse was the mean value of PC1 and PC2, respectively, in the corresponding group. The ellipse was rotated to the direction of largest variation of the corresponding group. Beta set-significance analysis showed no significant differences in genera between the preand post-treatment groups. In the ND groups, the main genera found were Staphylococcus (22.08\% in pre-ND and $26.33 \%$ in post-ND) and Cutibacterium (11.46\% in pre-ND and $13.70 \%$ in post-ND) in all subjects analyzed. The main genera found in the NF groups were also Staphylococcus $(12.518 \%$ in pre-ND and $13.19 \%$ in post-ND) and Cutibacterium (11.62\% in pre-ND and $11.45 \%$ in post-ND). There was a significant decrease in the relative abundance of the Pediococcus genus between the pre-NF and post-NF groups $(p<0.05)$ but no significant difference between the pre-ND and post-ND groups. Moreover, there were significant species differences between the pre-NF and post-NF groups $(p<0.05)$. In the pre-ND and post-ND groups, S. aureus $(22.10 \%$ and $26.27 \%$, respectively) and Cutibacterium acnes (formerly called P. acnes; $11.15 \%$ and $13.48 \%$, respectively) were most predominant (Figure 5). In particular, the abundance of S. aureus, which is known to be a pro-inflammatory bacterium, in the post-NF group was significantly decreased compared with that in the post-ND group $(p<0.05)$. Among the major genera belonging to Firmicutes, the abundance of Staphylococcus was not significantly different when compared between the pre-ND and pre-NF groups or the pre-NF and post-NF groups; however, when comparing the post-ND and post-NF groups, significant differences were found 
following the application of GOS-containing cosmetic serum ( $p<0.05$, Figure 5 and Supplementary 3$)$. In contrast, no significant differences were observed in the relative abundance of the main genus belonging to the other phylum groups. Cutibacterium and Enterobacteriaceae_g populations decreased, and Burkholderia, Thermoanaerobacterium, and Sphingomonas populations increased in the post-NF group but were not significantly differently compared with that in the post-ND group. The relative abundance of total lactic acid bacteria in the pre-treatment groups was slightly higher than that in the post-treatment groups (Figure 6). Conversely, the relative abundance of total lactic acid bacteria in the post-NF group was higher than that in the post-ND group but the difference was not significant. This observation suggests that the serum base lowers lactic acid abundance and that GOS supplementation increases the population of lactic acid bacteria (Figure 5A). Furthermore, the Lactococcus population was higher in the post-treatment groups than that in the pre-treatment groups; in particular, a significant increase was observed in the post-ND group compared with that in the pre-ND group ( $p<0.05$, Figure $6 \mathrm{~B}$ ). The relative abundance of Lactococcus increased in the post-NF group but was not significantly different. Further, the relative abundance of Pediococcus was significantly higher in the pre-treatment groups than that in the post-treatment groups $(p<0.05)$ and in the post-NF group than that in the post-ND group $(p<0.05)$.
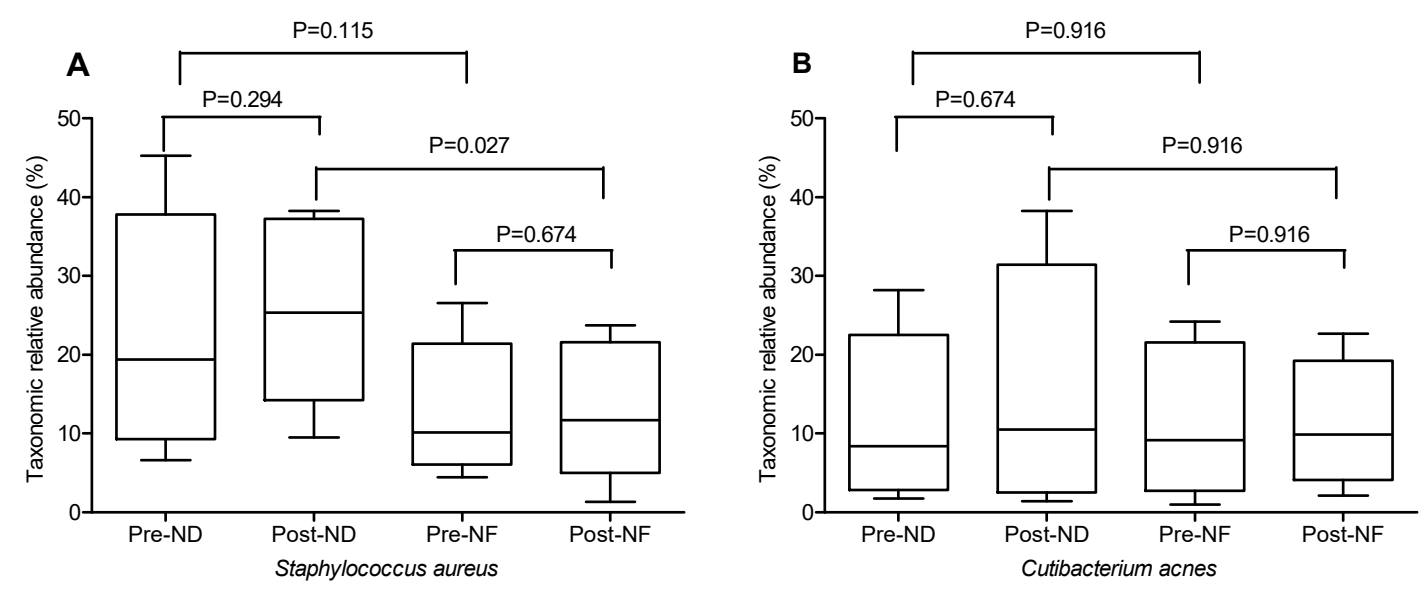

Figure 5. The relative abundances of Staphylococcus aureus $(\mathbf{A})$ and Cutibacterium acnes $(\mathbf{B})$ in the four groups. ND (control serum); NF (cosmetic serum containing GOS).
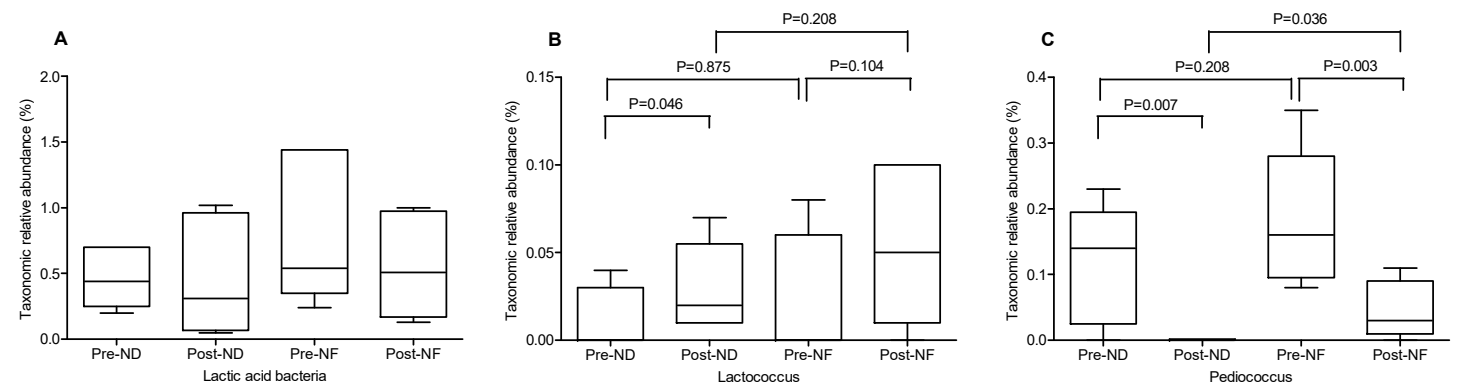

Figure 6. The relative abundances of Lactic acid bacteria (A), Lactococcus (B) and Pediococcus (C) in the four groups. ND (control serum); NF (cosmetic serum containing GOS).

\subsection{Correlation Analyses between Skin Microbiota and Skin Parameters}

Pearson's correlation analyses were performed on the top 10 relative abundant microbial genera and skin-related parameters (Figure 7). In the ND group, water-holding capacity and Enhydrobacter were significantly positively correlated, compared with the other bacteria. In the NF group, however, there was a negative correlation suggesting that Enhydrobacter growth was inhibited by the GOS-induced 
growth of lactic acid bacteria. In fact, the Enhydrobacter population decreased from $1.53 \%$ to $0.56 \%$ following GOS-containing cosmetic serum treatment. Further, in the ND group, TEWL was a significantly positively correlated with Bacillus, and Staphylococcus was negatively correlated with the melanin and erythema indexes. Conversely, in the NF group, Staphylococcus was positively correlated with melanin and erythema indexes. Moreover, in the NF group, there was no significant correlation between water-holding capacity and microorganisms; but there was a negative correlation between Enterobacteriaceae and TEWL.

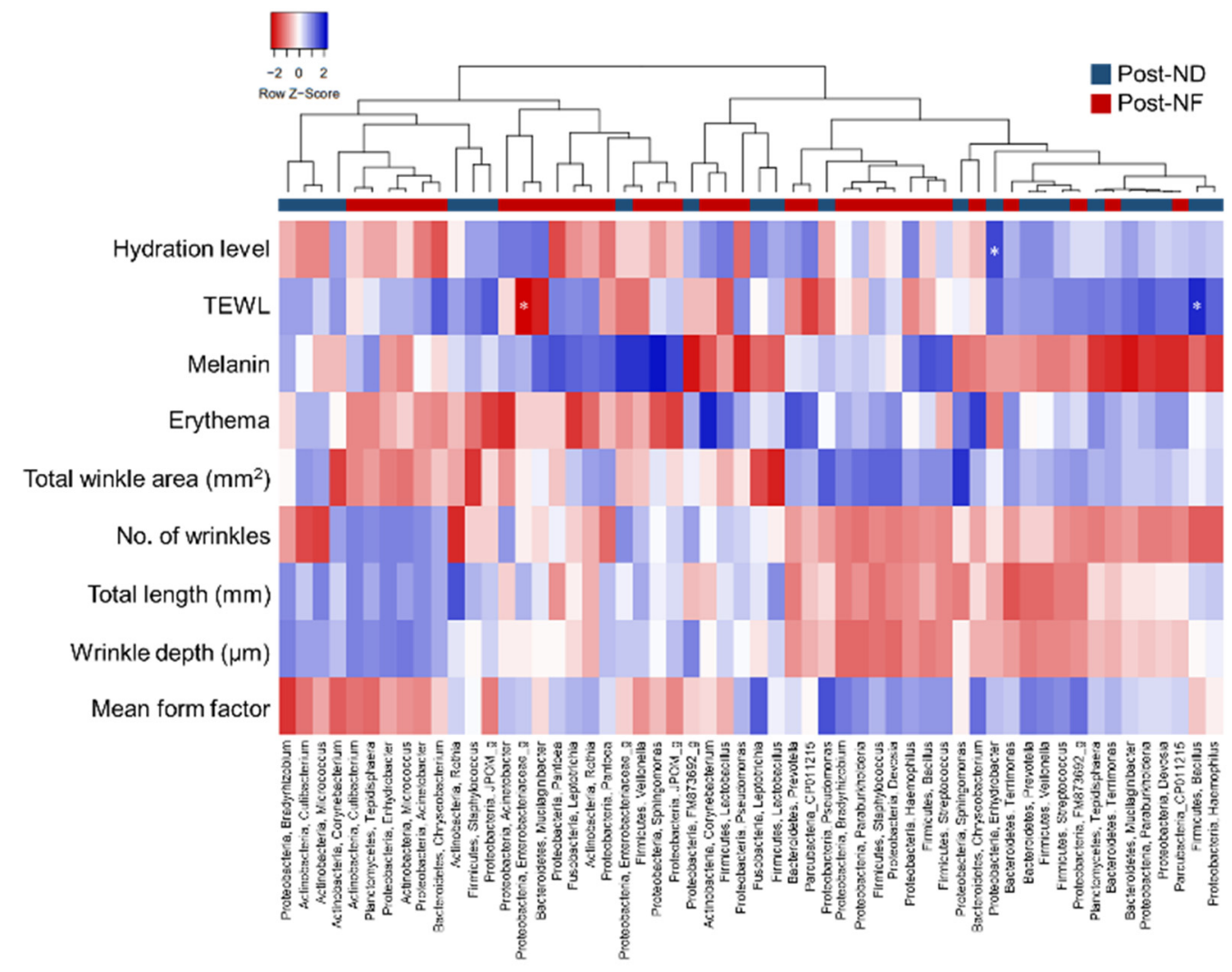

Figure 7. Pearson's correlation analyses of skin microbiota with clinical parameters after treatment of serum cosmetics with/without GOS. Correlations of significant genes expression in ND and NF group with clinical parameters and skin microbiota are shown by using heat map. * Pearson's correlation values with $p<0.05$. ND (control serum); NF (cosmetic serum containing GOS).

\section{Discussion}

Skin microbial communities differ from one individual to another owing to the genetic and environmental factors. Skin conditions, such as $\mathrm{pH}$, temperature, and moisture content, and a variety of factors, including antibiotics, cosmetics, soaps, personal hygiene products, lifestyle, and nutrients, affect the skin microbial community and abundance. Application of probiotics and prebiotics to the skin causes specific changes to the microbial diversity and colonization, leading to reconstitution of skin microbial communities. The re-balance of skin microbiota may have a significant effect on the functional differences between healthy and damaged skin. Therefore, we evaluated the potential of GOS, a prebiotic, as a cosmetic material by measuring the changes of skin factors and skin microorganisms.

Oral ingestion of GOS has been reported to be effective in moisturizing, maintaining skin barrier function, inhibiting pigmentation [22], and improving wrinkles [23]. Unlike previous studies, GOS-containing cosmetic serum were applied to the skin in this experiment and found a significant difference in water-holding capacity and TEWL, corresponding to the skin barrier function, compared with those in the ND group (Figures 1 and 2). The stratum corneum of the epidermis, the outermost layer of the skin, acts as a barrier to the harmful external environment and plays a vital role in preventing 
and retaining moisture in the skin [24]. The stratum corneum of the epidermis, the outermost layer of the skin, acts as a barrier to the harmful external environment and plays a vital role in preventing and retaining moisture in the skin [25], keeping the skin moist and shiny. When a lactic acid bacteria culture was applied to the skin, it maintained the $\mathrm{pH}$ at weakly acidic and contributed to skin hydration $[24,26]$. In the present study, application of GOS-containing cosmetic serum led to the growth of lactic acid bacteria among the skin microorganisms, which may have a moisturizing effect. The lactic acid bacteria such as Streptococcus pneumoniae (Supplementary 4) were found to be increased in the NF group compared with that in the ND group. There was no significant difference in S. pneumoniae levels in pre-NF and post-NF treatment group (NF) in serum treatment group containing GOS. In addition, the serum treatment group without GOS (post-ND) also showed no significant difference in S. pneumoniae level when compared to the post-NF group (Supplementary 5). S. aureus and S. pneumoniae are common pathogens associated with minor and invasive infections in the skin. Serum treatment containing GOS was effective in reducing S. aureus among pathogenic microorganisms on the skin. Additionally, oral administration of GOS increases the mRNA expression of tissue inhibitors of metalloproteinase (TIMP-1), which is an inhibitor of the collagen-degrading enzyme matrix metalloproteinase (MMP). In addition, GOS and Bifidobacterium co-administration reported a decrease in MMP-9 expression [27]. The GOS-containing serum treatment induced the proliferation of Lactobacillus, showing the possibility of suppressing MMP expression. The most abundant phyla identified on the skin of the subjects were Proteobacteria, Firmicutes, and Actinobacteria (Supplementary 2), corroborating with previous studies. Staphylococcus is the most common among the Firmicutes phylum, while Cutibacterium is the most abundant species among the Actinobacteria phylum.

Among the skin microorganisms of the subjects participating in the experiment, Proteobacteria, Firmicutes, and Actinobacteria were identified as the most abundant phyla (Supplementary 2). In other studies, these three phyla have been reported to be the most prevalent on the skin [28,29]. Staphylococcus is the most common among Firmicutes phylum, while actinobacteria is the most abundant species among Cutibacterium. The facial skin samples from the pre-treatment groups harbored fewer species than those from the post-treatment groups. Moreover, no statistically significant difference was observed in the bacterial richness on the facial skin of the post-NF group compared with the post-ND group; however, the diversity of the bacterial communities was found to be caused by the application of the GOS-containing cosmetic serum (Figure 4). GOS treatment was expected to induce higher diversity than the serum base treatment because GOS as a prebiotic enhances environmental diversity of the skin microbes. Prebiotics are "fertilizers" or "foods" with components that selectively promote the growth of these essential microorganisms, thereby potentially improving the host health [30]. S. aureus was the most common species found on the skin of the subjects, as shown in Supplementary 3 and Figure 5, and has been recognized as a crucial cause of skin infection [31]. Staphylococcus is relatively common on healthy skin but in one-third of the population the presence of $S$. aureus is considered a significant risk factor for future infections [32]. S. aureus weakens the skin barrier and activates deleterious host immune responses. For instance, $S$. aureus produces proteases that are capable of penetrating the dermis of patients or mice with atopy disease $(\mathrm{AD})$ and deleterious mutations in the filaggrin gene $[33,34]$. In fact, in $\mathrm{AD}$, abundance of $S$. aureus was associated with immune dysfunctions, including T helper cell 2 lymphocyte asymmetry, reduced antimicrobial peptides (AMPs), exacerbated allergic reactions, and destruction of the skin barrier [35]. S. aureus increases the production of type 2 cytokines, such as thymic stromal lymphopoietin, interleukin (IL)-4, and IL-13 [33]. Moreover, several other molecules produced by $S$. aureus also induce skin inflammation and exacerbate AD. In particular, $\alpha$-toxin degrades keratinocytes in the presence of type 2 cytokines. All these factors contribute to local inflammation and further affect the skin barrier, leading to the exacerbation of skin diseases such as AD [36]. However, other bacterial species found on normal skin seem to help maintain immune homeostasis [37]. S. epidermidis present on the skin of healthy individuals may reduce inflammation [38], improve the development of skin T cells, and promote the expression of AMPs [39]. 
Skin sites can be classified based on their physiological properties as sebaceous (oily), moist, or dry and can affect the distribution of the skin microbiota [2]. Enhydrobacter, Corynebacterium, and Staphylococcus are predominant at the moist sites. In the ND group, water-holding capacity was positively correlated with these strains (Figure 7). Moreover, TEWL was positively correlated with the relative abundance of S. aureus but was not significantly different (Figure 7). In the human AD epidermis, the relative abundance of $S$. aureus and S. epidermis is increased while that of Propionibacterium decreased relative to other genera (Streptococcus, Acinetobacter, Corynebacterium, and Prevotella). Furthermore, in this study, Bacillus and TEWL were significantly positively correlated in the ND group. Bacillus is a genus belonging to Firmicutes and is found on normal skin [40]. Further, increased colonization of Enterobacteriaceae is observed in adult and infant populations with AD [41,42]. In fact, in the case of AD, TEWL is reduced, demonstrating a negative correlation between Enterobacteriaceae and TEWL.

In our previous study [22,43], GOS were shown to inhibit the production of pro-inflammatory agents IL-6, IL-8, and IL-12; tumor necrosis factor- $\alpha$; and prostaglandin E2 in UV-irradiated HaCaT cells and hairless mice. The increase in the population of lactic acid bacteria and inhibition of inflammation-producing substances by GOS may have reduced the population of S. aureus and production of pro-inflammatory substances. The application of cosmetics containing prebiotics from certain plant extracts is effective in inhibiting the growth of the inflammation-causing bacterium but not that of coagulase-negative Staphylococci [8]. The application of prebiotics is far superior to that of antimicrobial cosmetics, which use antibiotics or antibacterial agents to unselectively reduce bacterial growth [44]. Moreover, prebiotics such as GOS improve the host health by selectively stimulating the growth of beneficial bacterial species such as Bifidobacteria and Lactobacilli. Additionally, various lactic acid bacteria, including Lactobacillus, Lactococcus, Pediococcus, Propionibacterium, Leuconostoc, and Carnobacterium have been reported to use prebiotics for the production of bacteriocins $[45,46]$. Oh et al. [46] reported a clinical test which revealed that bacteriocin HY 449 from Lactococcus spp. regulates skin inflammation and acne and inhibits the growth of inflammatory skin bacteria, such as S. aureus, Streptococcus pyogenes, and P. acnes. The production of bacteriocin, owing to GOS-induced lactic acid secretion, may also reduce the number of harmful microorganisms on the skin.

\section{Conclusions}

In conclusion, a randomized controlled trial was used to evaluate skin-related parameters in healthy adults who treated with GOS-containing cosmetic serum for 8 weeks. This study supports the principal concept that the application of GOS-containing serum can be used for the management of the composition and diversity of the human skin microbiota. Treatment with GOS-containing cosmetic serum is effective in improving various skin parameters and the population of beneficial skin microbes. However, since the analysis of the skin microbial community was performed in a small number of subjects, additional experiments are needed to investigate the correlation between the application of GOS and the growth of harmful microorganisms such as Streptococcus spp. at various ages and subjects.

Supplementary Materials: The following are available online at http://www.mdpi.com/2075-4426/10/3/91/s1, Figure S1: The relative abundance of the most prevalent bacterial groups associated with each microenvironment depicted for all the subjects investigated. Superscripts indicate phylum; Figure S2: The relative abundance of the most prevalent bacterial groups in each group; Table S1: The relative abundance of the main genera of bacteria in post-treatment groups (post-ND and post-NF); Table S2: The relative abundance of the main species of bacteria in post-treatment groups (post-ND and post-NF).

Author Contributions: Conceptualization, H.J.S. and E.Y.J.; data curation, K.-B.H. and K.J.; formal analysis. Y.H.H. and K.J.; funding acquisition, H.J.S.; investigation, K.-B.H. and K.J.; methodology, K.-B.H., K.J., and H.J.S.; project administration, K.-B.H., Y.H.H., E.Y.J., K.J., and H.J.S.; resources, K.-B.H., K.J., and H.J.S.; software, K.-B.H. and H.J.S.; supervision, H.J.S.; validation, Y.H.H., E.Y.J., and H.J.S.; visualization, K.-B.H. and H.J.S.; writing-original draft, K.-B.H., K.J. and H.J.S. All authors have read and agreed to the published version of the manuscript.

Funding: This work was supported by the Technology development program (S2611313) funded by the Ministry of SMEs and Startups (MSS, Korea).

Conflicts of Interest: The authors declares no conflict of interest. 


\section{References}

1. Dabrowska, A.; Spano, F.; Derler, S.; Adlhart, C.; Spencer, N.; Rossi, R. The relationship between skin function, barrier properties, and body-dependent factors. Ski Res. Technol. 2018, 24, 165-174. [CrossRef]

2. Byrd, A.L.; Belkaid, Y.; Segre, J.A. The human skin microbiome. Nat. Rev. Microbiol. 2018, 16, $143-155$. [CrossRef]

3. Cundell, A.M. Microbial Ecology of the Human Skin. Microb. Ecol. 2018, 76, 113-120. [CrossRef]

4. Chen, Y.E.; Fischbach, M.A.; Belkaid, Y. Skin microbiota-host interactions. Nature 2018, 553, 427-436. [CrossRef] [PubMed]

5. Rosenthal, M.; Goldberg, D.; Aiello, A.; Larson, E.; Foxman, B. Skin microbiota: Microbial community structure and its potential association with health and disease. Infect. Genet. Evol. 2011, 11, 839-848. [CrossRef] [PubMed]

6. Wyatt, M.A.; Wang, W.; Roux, C.M.; Beasley, F.C.; Heinrichs, D.E.; Dunman, P.M.; Magarvey, N.A. Staphylococcus aureus nonribosomal peptide secondary metabolites regulate virulence. Science 2010, 329, 294-296. [CrossRef] [PubMed]

7. Secor, P.R.; Jennings, L.K.; James, G.A.; Kirker, K.R.; Pulcini, E.D.; McInnerney, K.; Gerlach, R.; Livinghouse, T.; Hilmer, J.K.; Bothner, B.; et al. Phevalin (aureusimine B) production by Staphylococcus aureus biofilm and impacts on human keratinocyte gene expression. PLoS ONE 2012, 7, e40973. [CrossRef]

8. Bockmühl, D.; Jassoy, C.; Nieveler, S.; Scholtyssek, R.; Wadle, A.; Waldmann-Laue, M. Prebiotic cosmetics: An alternative to antibacterial products. Int. J. Cosmet. Sci. 2007, 29, 63-64. [CrossRef]

9. Ouwehand, A.; Lahtinen, S.; Tiihonen, K. The Potential of Probiotics and Prebiotics for Skin Health. In Textbook of Aging Skin; Springer: Berlin/Heidelberg, Germany, 2010; pp. 799-810.

10. Davani-Davari, D.; Negahdaripour, M.; Karimzadeh, I.; Seifan, M.; Mohkam, M.; Masoumi, S.J.; Berenjian, A.; Ghasemi, Y. Prebiotics: Definition, Types, Sources, Mechanisms, and Clinical Applications. Foods 2019, 8, 92. [CrossRef]

11. Hong, Y.H.; Chang, U.J.; Kim, Y.S.; Jung, E.Y.; Suh, H.J. Dietary galacto-oligosaccharides improve skin health: A randomized double blind clinical trial. Asia Pac. J. Clin. Nutr. 2017, 26, 613-618. [CrossRef]

12. Di Mauro, M.D.; Tomasello, B.; Giardina, R.C.; Dattilo, S.; Mazzei, V.; Sinatra, F.; Caruso, M.; D’Antona, N.; Renis, M. Sugar and mineral enriched fraction from olive mill wastewater for promising cosmeceutical application: Characterization, in vitro and in vivo studies. Food Funct. 2017, 8, 4713-4722. [CrossRef] [PubMed]

13. Pillai, R.; Redmond, M.; Röding, J. Anti-wrinkle therapy: Significant new findings in the non-invasive cosmetic treatment of skin wrinkles with beta-glucan. Int. J. Cosmet. Sci. 2005, 27, 292. [CrossRef]

14. Fitzpatrick, T.B. The Validity and practicality of sun-reactive skin type-I through type-Vi. Arch. Dermatol. 1988, 124, 869-871. [CrossRef] [PubMed]

15. Caporaso, J.G.; Kuczynski, J.; Stombaugh, J.; Bittinger, K.; Bushman, F.D.; Costello, E.K.; Fierer, N.; Pena, A.G.; Goodrich, J.K.; Gordon, J.I.; et al. QIIME allows analysis of high-throughput community sequencing data. Nat. Methods 2010, 7, 335-336. [CrossRef]

16. Quince, C.; Lanzen, A.; Curtis, T.P.; Davenport, R.J.; Hall, N.; Head, I.M.; Read, L.F.; Sloan, W.T. Accurate determination of microbial diversity from 454 pyrosequencing data. Nat. Methods 2009, 6, 639-641. [CrossRef]

17. Soergel, D.A.W.; Dey, N.; Knight, R.; Brenner, S.E. Selection of primers for optimal taxonomic classification of environmental 16S rRNA gene sequences. ISME J. 2012, 6, 1440-1444. [CrossRef]

18. Cole, J.R.; Wang, Q.; Cardenas, E.; Fish, J.; Chai, B.; Farris, R.J.; Kulam-Syed-Mohideen, A.S.; McGarrell, D.M.; Marsh, T.; Garrity, G.M.; et al. The ribosomal database project: Improved alignments and new tools for rRNA analysis. Nucleic Acids Res. 2009, 37, D141-D145. [CrossRef]

19. Schloss, P.D.; Westcott, S.L.; Ryabin, T.; Hall, J.R.; Hartmann, M.; Hollister, E.B.; Lesniewski, R.A.; Oakley, B.B.; Parks, D.H.; Robinson, C.J.; et al. Introducing mothur: Open-source, platform-independent, community-supported software for describing and comparing microbial communities. Appl. Environ. Microbiol. 2009, 75, 7537-7541. [CrossRef]

20. Mount, D.W. Using the basic local alignment search tool (BLAST). Cold Spring Harb. Protoc. 2007, 2007. [CrossRef]

21. Houser, T.; Zerweck, C.; Grove, G.; Wickett, R. Shadow analysis via the C+ K Visioline: A technical note. Ski Res. Technol. 2017, 23, 447-451. [CrossRef] 
22. Suh, M.G.; Hong, Y.H.; Jung, E.Y.; Suh, H.J. Inhibitory Effect of Galactooligosaccharide on Skin Pigmentation. Prev. Nutr. Food Sci. 2019, 24, 321-326. [CrossRef] [PubMed]

23. Jung, E.Y.; Kwon, J.I.; Hong, Y.H.; Suh, H.J. Evaluation of Anti-Wrinkle Effects of DuOligo, Composed of Lactulose and Galactooligosaccharides. Prev. Nutr. Food Sci. 2017, 22, 381-384. [CrossRef] [PubMed]

24. Alberts, B.; Bray, D.; Lewis, J.; Raff, M.; Roberts, K.; Watson, J. Molecular Biology of the Cell; Biochemistry and Molecular Biology Education: Garland, NY, USA, 1994; pp. 907-982.

25. Verdier-Sévrain, S.; Bonté, F. Skin hydration: A review on its molecular mechanisms. J. Cosmet. Dermatol. 2007, 6, 75-82. [CrossRef] [PubMed]

26. Izawa, N.; Sone, T. Cosmetic ingredients fermented by lactic acid bacteria. In Microbial Production; Springer: Berlin, Germany, 2014; pp. 233-242.

27. Hong, K.-B.; Jeong, M.; Han, K.S.; Hwan Kim, J.; Park, Y.; Suh, H.J. Photoprotective effects of galacto-oligosaccharide and/or Bifidobacterium longum supplementation against skin damage induced by ultraviolet irradiation in hairless mice. Int. J. Food Sci. Nutr. 2015, 66, 923-930. [CrossRef]

28. Dekio, I.; Hayashi, H.; Sakamoto, M.; Kitahara, M.; Nishikawa, T.; Suematsu, M.; Benno, Y. Detection of potentially novel bacterial components of the human skin microbiota using culture-independent molecular profiling. J. Med. Microbiol. 2005, 54, 1231-1238. [CrossRef]

29. Gao, Z.; Perez-Perez, G.I.; Chen, Y.; Blaser, M.J. Quantitation of major human cutaneous bacterial and fungal populations. J. Clin. Microbiol. 2010, 48, 3575-3581. [CrossRef]

30. La Fata, G.; Rastall, R.A.; Lacroix, C.; Harmsen, H.J.M.; Mohajeri, M.H.; Weber, P.; Steinert, R.E. Recent Development of Prebiotic Research-Statement from an Expert Workshop. Nutrients 2017, 9, 1376. [CrossRef]

31. von Eiff, C.; Becker, K.; Machka, K.; Stammer, H.; Peters, G.; Grp, S. Nasal carriage as a source of Staphylococcus aureus bacteremia. N. Engl. J. Med. 2001, 344, 11-16. [CrossRef]

32. Weidenmaier, C.; Goerke, C.; Wolz, C. Staphylococcus aureus determinants for nasal colonization. Trends Microbiol. 2012, 20, 243-250. [CrossRef]

33. Nakatsuji, T.; Chen, T.H.; Two, A.M.; Chun, K.A.; Narala, S.; Geha, R.S.; Hata, T.R.; Gallo, R.L. Staphylococcus aureus Exploits Epidermal Barrier Defects in Atopic Dermatitis to Trigger Cytokine Expression. J. Investig. Dermatol. 2016, 136, 2192-2200. [CrossRef]

34. Hirasawa, Y.; Takai, T.; Nakamura, T.; Mitsuishi, K.; Gunawan, H.; Suto, H.; Ogawa, T.; Wang, X.L.; Ikeda, S.; Okumura, K.; et al. Staphylococcus aureus Extracellular Protease Causes Epidermal Barrier Dysfunction. J. Investig. Dermatol. 2010, 130, 614-617. [CrossRef] [PubMed]

35. Kemter, A.M.; Nagler, C.R. Influences on allergic mechanisms through gut, lung, and skin microbiome exposures. J. Clin. Investig. 2019, 129, 1483-1492. [CrossRef] [PubMed]

36. Nakatsuji, T.; Chen, T.H.; Narala, S.; Chun, K.A.; Two, A.M.; Yun, T.; Shafiq, F.; Kotol, P.F.; Bouslimani, A.; Melnik, A.V.; et al. Antimicrobials from human skin commensal bacteria protect against Staphylococcus aureus and are deficient in atopic dermatitis. Sci. Transl. Med. 2017, 9. [CrossRef] [PubMed]

37. Gallo, R.L.; Nakatsuji, T. Microbial Symbiosis with the Innate Immune Defense System of the Skin. J. Investig. Dermatol. 2011, 131, 1974-1980. [CrossRef]

38. Lai, Y.; Di Nardo, A.; Nakatsuji, T.; Leichtle, A.; Yang, Y.; Cogen, A.L.; Wu, Z.R.; Hooper, L.V.; Schmidt, R.R.; von Aulock, S.; et al. Commensal bacteria regulate Toll-like receptor 3-dependent inflammation after skin injury. Nat. Med. 2009, 15, 1377-1382. [CrossRef] [PubMed]

39. Wanke, I.; Steffen, H.; Christ, C.; Krismer, B.; Gotz, F.; Peschel, A.; Schaller, M.; Schittek, B. Skin commensals amplify the innate immune response to pathogens by activation of distinct signaling pathways. J. Investig. Dermatol. 2011, 131, 382-390. [CrossRef] [PubMed]

40. Ramadan, M.; Solyman, S.; Yones, M.; Halaby, H.; Abdalla, Y.; Hanora, A. Shotgun Metagenomic analysis of cutaneous microbiome in patients with atopic dermatitis. Rec. Pharm. Biomed. Sci. 2019, 3, 1-3. [CrossRef]

41. Matsumoto, M.; Ohishi, H.; Kakizoe, K.; Benno, Y. Faecal microbiota and secretory immunogloblin a levels in adult patients with atopic dermatitis. Microb. Ecol. Health Dis. 2004, 16, 13-17.

42. Yap, G.C.; Loo, E.X.L.; Aw, M.; Lu, Q.; Shek, L.P.-C.; Lee, B.W. Molecular analysis of infant fecal microbiota in an Asian at-risk cohort-correlates with infant and childhood eczema. BMC Res. Notes 2014, 7, 166. [CrossRef]

43. Suh, M.G.; Bae, G.Y.; Jo, K.; Kim, J.M.; Hong, K.B.; Suh, H.J. Photoprotective Effect of Dietary Galacto-Oligosaccharide (GOS) in Hairless Mice via Regulation of the MAPK Signaling Pathway. Molecules 2020, 25, 1679. [CrossRef] 
44. Holland, K.T.; Bojar, R.A. Cosmetics. Am. J. Clin. Dermatol. 2002, 3, 445-449. [CrossRef] [PubMed]

45. Klaenhammer, T.R. Genetics of bacteriocins produced by lactic-acid bacteria. FEMS Microbiol. Rev. 1993, 12, 39-86. [CrossRef]

46. Oh, S.; Kim, S.H.; Ko, Y.; Sim, J.H.; Kim, K.S.; Lee, S.H.; Park, S.; Kim, Y.J. Effect of bacteriocin produced by Lactococcus sp HY 449 on skin-inflammatory bacteria. Food Chem. Toxicol. 2006, 44, 1184-1190. [CrossRef] [PubMed]

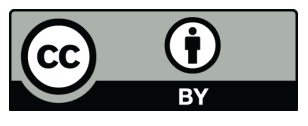

(C) 2020 by the authors. Licensee MDPI, Basel, Switzerland. This article is an open access article distributed under the terms and conditions of the Creative Commons Attribution (CC BY) license (http://creativecommons.org/licenses/by/4.0/). 